the baby's interests. The welfare of any child is inextricably bound up with the interests of their family. The baby's interests take priority because it is their very life that is at stake. However, the baby's interests cannot be properly assessed without taking some account of others' interests too.

\section{NOT FAR ENOUGH?}

Uncertainty of outcome may lead doctors and parents to agree to start intensive care. The baby does not do well. Doctors conclude that after all their prospects of survival are poor and/or there are serious, irremediable impairments. Continuing invasive care is no longer in the baby's best interests. The report concludes that there is no moral distinction between never instituting intensive care and later withdrawing such care. Yet we unreservedly reject the precedent set in the Netherlands in the Groeningen Protocol. Doctors should not be permitted to take active steps to end newborn life. Wrong headed and inconsistent, argue both proponents of neonatal euthanasia and some opponents of abortion. If obstetricians can end fetal life up to delivery, how is neonaticide different?

The working party endorsed birth as a significant threshold for moral as well as for legal judgments. We did so while acknowledging that many people will see human life as starting much earlier. But for our part (whatever our personal views on fetal status) birth marks the point at which a baby enters the world and is separate from its mother, and can be cared for without intruding on the mother's bodily integrity.

Some people will see deliberately ending a life as no different morally from allowing it to end. For those critics, we should have followed the Dutch example and permitted active steps to end the life of the newborn; for others what we recommend is already just as bad as the Dutch rules and should be condemned out of hand for that alone.

Doctors and parents do see a real and huge difference between ending treatment that only prolongs a terrible life, and giving a lethal injection. We felt it right to respect the feelings of those most intimately involved in decisions about premature babies and who may want the opportunity to spend time caring for a dying baby. And there is a problem of consistency-if we can take a decision to end the life of a baby why should we not also take decisions to end the lives of older children or adults who cannot speak for themselves? Allowing neonatal euthanasia opens the door to non-voluntary euthanasia.
We suspect that a report which attracts such diverse views is probably doing something right. We have tried to start an honest debate about these issues. If premature babies, parents and doctors benefit in the long term, then, and only then, will we know that the report has achieved its objectives.

J Med Ethics 2007;33:125-126. doi: 10.1136/jme.2006.020099

\section{Authors' affiliations}

Margaret Brazier, School of Law, University of Manchester, Manchester, UK

David Archard, The Institute for Philosophy and Public Policy, Lancaster University, Lancaster, California, USA

Correspondence to: Professor M Brazier, School of Law, University of Manchester, Oxford Road, Manchester M13 9PL, UK; margaret.brazier@ manchester.ac.uk

Received 5 December 2006

Revised 5 December 2006

Accepted 5 December 2006

Competing interests: None.

\section{REFERENCE}

1 http://www.nuffieldbioethics.org laccessed 12 January 2007, The authors were respectively the Chair and a Member of the Working Party that drafted the report).

the appropriateness of resuscitation, itself depend on the initiation of resuscitation? To what extent is it reasonable and

\title{
End of life decision-making in neonatal
} care

\section{Carolyn April, Michael Parker}

\section{Critical care of neonates}

$\mathrm{T}$ he recently published report of the Nuffield Council on Bioethics, Critical care decisions in fetal and neonatal medicine, is a valuable contribution to the discussion of decision making in the critical care of neonates. Drawing upon medical evidence, the working party highlights the many practical difficulties arising in neonatal care and by setting out clearly the nature of the ethical and other issues arising in this area of medicine, and their relationship with neonatal development, the resulting report has the potential to lead both to improved practice and to better informed communication between doctors and families when they face difficult decisions about how best to treat very premature babies. Based on medical evidence, the working party sets out guidelines on decision-making about the resuscitation of babies born before the gestational age of 25 weeks and 6 days, dividing this period into four chronological periods: before 21 weeks and 6 days when resuscitation should normally only take place within the context of a research project; between 22 weeks and 23 weeks when resuscitation should not normally be carried out unless the parents request it; at 23 or 24 weeks when parental views should take precedence; and, after 24 weeks when resuscitation should be the norm unless not in the child's best interests.

There are several practical ethical difficulties with this aspect of the advice. To what extent, for example, does the paediatric assessment of the child, intended to play a role in decision-making about humane to expect parents to take full responsibility for making decisions about resuscitation between 23 and 24 weeks? On what grounds is it acceptable to attempt to resuscitate a baby $<21$ weeks and 6 days for a research project, but not when the parents request it for other reasons? Nevertheless, despite these difficulties, and although some members of the British Medical Association have attacked the guidelines as too restrictive and undermining of professional judgement, they have the potential, in our view, to lead to the development of a greater degree of agreed good practice and thereby to constitute an important contribution to neonatal care.

Judging by the media coverage of the report, the issue attracting the most attention as well as the most controversy concerns the ethics of active euthanasia in neonatal care. This controversy arises in part from a request by the Royal College of Obstetricians and Gynaecologists, London, UK that the working party present a discussion on whether active euthanasia should ever play a role in neonatal critical care. The report does take a strong position on the issue and "unreservedly rejects" the 
precedent set in the Netherlands' Groningen Protocol, which holds that active euthanasia is morally permissible in some neonatal critical care cases. Despite the working party's uncompromising stance, the report does not really constitute the kind of sustained and deliberative engagement with the ethics of active euthanasia envisaged by the Royal College of Obstetricians and Gynaecologists, and in this respect constitutes a missed opportunity.

In rejecting active euthanasia as morally impermissible, the report maintains that in terminal cases there is an obligation to provide palliative care to suffering newborns. However, the report also cites evidence that most healthcare professionals working in neonatal intensive care do not receive any mandatory training in palliative care and that access to care givers who are specially trained in palliative care is extremely limited. This suggests that many neonates who would benefit will not in fact have access to appropriate palliative care, and implies too that the morally significant question of what care givers' obligations are to the suffering newborn if and when palliative care fails to relieve suffering is unavoidable. If the duty to provide palliative care arises from a more general duty to relieve suffering, then on what grounds can the only remaining option for relieving a newborn's suffering in such cases, namely a quick and painless end to life, be held to be morally impermissible?

In support of its position, the working party argues that although no morally relevant distinction can be said to exist between withholding treatment and withdrawing treatment, such a distinction does exist between withholding or withdrawing treatment on the one hand and actively bringing about death on the other. The reasons the working party provides for the moral relevance of this distinction are: first, that the professional guidelines call for the distinction; second, that the working party's position reflects the current consensus in the UK and third that most doctors think this distinction exists. Setting aside questions on the empirical validity of these claims, these are not in fact moral arguments but appeals to public opinion and the moral primacy of the status quo.

Although giving the impression of a solution, within the context of the argument set out in the report this appeal creates more problems than it solves because it rests on an inconsistency. For maintaining a distinction between withholding or withdrawing treatment on the one hand and active euthanasia on the other, the report appeals to the views of doctors, but, in maintaining the absence of a distinction between withholding and withdrawing treatment, it uses an appeal to overarching philosophical principles to dismiss what is in fact the opinion of many doctors and nurses-namely that there is a morally relevant distinction between withholding treatment and withdrawing treatment from a patient for where this has been initiated. Why should the doctors' opinions be relied on in one case but dismissed in the other?

To their credit, the working party does seem to acknowledge that many of the reasons it presents to deny the permissibility of euthanasia are not in fact moral reasons. However, when the report does go on to make explicit moral arguments, these are rather uninspiring. The notion of a slippery slope is called upon, for example, to claim that allowing neonatal euthanasia would open the door to adult non-voluntary euthanasia. But, for this argument to work, three other claims must also hold: firstly, that adult non-voluntary euthanasia is not morally permissible; secondly, that there are no morally relevant differences between newborns and adults in persistent vegetative states; and thirdly that no effective regulatory or other mechanism is available to maintain the distinction. Instead of seizing the opportunity to examine these claims, the report claims that the burden of proof to show morally relevant distinctions falls on those who wish to argue against the report's position.

In its second moral argument, the report claims that a philosophical principle known as the Doctrine of Double Effect renders deliberate killing morally impermissible, even in the case of suffering newborns for whom palliative care has failed. As is well known, the Doctrine of Double Effect holds that actions that have one bad outcome and one good outcome may be morally permissible if the good outcome is intended while the bad outcome is not intended, even if foreseen. The working party uses the Doctrine of Double Effect to argue that it is morally permissible for doctors to administer palliative treatment that hastens a newborn's death, but impermissible for them to administer a lethal injection to bring about death. What makes the moral difference between these two cases, the report claims, is that in the case of administering pain relief medication, the doctor merely foresees that the treatment will hasten death, whereas in the case of lethal injection, the doctor necessarily intends to kill.

The Doctrine of Double Effect is a powerful conceptual tool that is useful in the realm of the law and in enabling those with strong moral principles to act with compassion; however, its use here in the context of neonatal care is not unproblematic. For it shifts the core moral issue from concern about the newborn's irremediable suffering, which is the fundamental ethical concern on which much of the report is based, to concern about the moral status of the doctor's intentions. In light of the report's statement that the newborn's interests are the most important ethical considerations in critical care decision making, an extended debate on whether a shift in moral focus from those interests to the doctor's intentions is appropriate could have proven fruitful and may have even, we contend, altered the working party's final position.

Their expertise and experience in the fields of bioethics, law and medicine, meant that the members of the working party were well-placed to tackle the practical and philosophically interesting ethical issues surrounding end of life decisionmaking in neonatal critical care. Their talents are ably shown in the significant contributions the report makes to better understanding of the issues arising in neonatal care, in facilitating communication between doctors and families and in furthering the goals of procedural justice in healthcare. The members of the working party were also exceptionally well-qualified to engage ethical issues arising in neonatal critical care, as much of the report makes clear. However, this means that it is rather disappointing that the opportunity was missed to enrich and challenge public discussion about the central issue arising from the report, notably: neonatal active euthanasia. To a certain extent, the working party can be said to have complied with the request of the Royal College of Obstetricians and Gynaecologists to set out a discussion on what role, if any, active euthanasia should play in neonatal critical care, but by appealing to public opinion and invoking what have become the standard arguments against active euthanasia, the working party skirted the central issue. As a result, an opportunity was missed to apply the talents and expertise of the working party members for examining the issue of the ethics of active euthanasia through the lens of neonatal critical care, and thereby to take the euthanasia debate to a more sophisticated and challenging level than that exemplified by much of the media debate following the publication of the report.

J Med Ethics 2007;33:126-127. doi: 10.1136/jme.2006.020107

\section{Authors' affiliations Carolyn April, Michael Parker, The Ethox Centre, University of Oxford, Oxford, UK}

Correspondence to: Professor M Parker, The Ethox Centre, University of Oxford, Gibson Building/Block 21, Radcliffe Infirmary, Woodstock Road, Oxford OX2 6HE, UK michael.parker@ethox.ox.ac.uk

Received 5 December 2006

Revised 5 December 2006

Accepted 5 December 2006

Competing interest: None. 